\title{
Blocking TIGIT/CD155 Signal Reverses CD8+T Cell Exhaustion and Enhances the Anti-Tumor Ability of Cervical Cancer
}

\section{Lu Liu}

shan dong da xue yi xue yuan: Shandong University Cheeloo College of Medicine aihong wang

shan dong da xue yi xue yuan: Shandong University Cheeloo College of Medicine

Xiaoli Liu

shan dong da xue yi xue yuan: Shandong University Cheeloo College of Medicine

Sai Han

shan dong da xue yi xue yuan: Shandong University Cheeloo College of Medicine

Yu Sun

shan dong da xue yi xue yuan: Shandong University Cheeloo College of Medicine

Junhua Zhang

shan dong da xue yi xue yuan: Shandong University Cheeloo College of Medicine

Lingyu Guo

shan dong da xue yi xue yuan: Shandong University Cheeloo College of Medicine

youzhong zhang ( $\nabla$ zhangyouzhong@sdu.edu.cn)

Shandong University Qilu Hospital

\section{Research Article}

Keywords: Immune checkpoint, TIGIT/CD155, CD8+T, Cervical Cancer, immuno therapy Introduction

Posted Date: February 28th, 2022

DOI: https://doi.org/10.21203/rs.3.rs-1353432/v1

License: (1) (1) This work is licensed under a Creative Commons Attribution 4.0 International License. Read Full License 


\section{Abstract}

ObjectiveVTIGIT/CD155 has attracted widespread attention as a new immune checkpoint and a potential target for cancer immunotherapy. In our study, we evaluated the role of TIGIT/CD155 checkpoints in the progression of cervical cancer.

Methods. Detect the expression of CD155 and TIGIT in cervical cancer tissues by flow cytometry, immunohistochemistry and gene expression profiling. In vivo and in vitro experiments have proved that blocking TIGIT/CD155 restores the ability of CD8+T cells to produce cytokines.

Methods.खDetect the expression of CD155 and TIGIT in cervical cancer tissues by flow cytometry, immunohistochemistry $(\mathrm{IHC})$ and gene expression profiling. In vivo and in vitro experiments have proved that blocking TIGIT/CD155 restores the ability of CD8+T cells to produce cytokines.Changes in NF-KB and ERK pathways detected by western blot (WB) after blocking TIGIT/CD155 signaling

Results $\searrow$ We found that the expression of TIGIT is elevated in patients with cervical cancer. The high expression of TIGIT in CD8 ${ }^{+} \mathrm{T}$ lymphocytes of cervical cancer patients promotes the failure of CD $8^{+} \mathrm{T}$ lymphocytes. In addition, CD155 is highly expressed in cervical cancer tissues and negatively correlated with the infiltration level of $\mathrm{CD}^{+} \mathrm{T}$ cells. We showed that phosphorylated TIGIT binds to CD155 to inhibit NF-KB and ERK activation by recruiting SHIP-1, leading to down-regulation of cytokine production. Inactivated $C D 8^{+} T$ cells, after blocking TIGIT, the inhibitory effect of SHIP-1 on CD8 ${ }^{+} T$ cells is weakened, and the activation of NF-KB and ERK is enhanced. In vivo and in vitro experiments have proved that blocking TIGIT/CD155 restores the ability of $C D 8^{+} T$ cells to produce cytokines. Injecting blocking antibody TIGIT in vivo could inhibit tumor growth and promote $C D 8^{+} T$ lymphocyte function. Combining blocking TIGIT and PD-1 further increased the effect of the blocking antibody TIGIT.

Conclusions $₫$ Our research shows that TIGIT/CD155 is a potential therapeutic target for cervical cancer.

\section{Introduction}

Cervical cancer ranks fourth in morbidity and mortality among female malignancies [1]. In 2018, there were 570,000 new patients and 311,000 deaths worldwide [2]. Vaccination against human papillomavirus (HPV) and cervical cancer screening effectively prevent cervical cancer [3]. Surgery is the most commonly used treatment for early cervical cancer, and it has achieved great success. Conventional treatment options for metastatic/recurrent cervical cancer include radiotherapy and chemotherapy, but this treatment has limited effect on advanced disease [4].Tumor cells can cause a robust immune response, but cancer still occurs. The immune response caused by tumor cells is insufficient to prevent cancer development, or a short-term immune response triggers a specific immune tolerance mechanism in tumor cells [5].A healthy immune system requires a balance of immune checkpoint blockade (ICB). In recent years, the development of immunomodulatory drugs that block the ICB pathway has become a promising strategy for treatingvarious malignant tumors. Anti-programmed cell death 1 (PD-1) mAband anti- 
cytotoxic T lymphocyte-associated antigen 4 (CTLA-4) mAbare therapeutic targets studied in a variety of malignant tumors, including cervical cancer [6,7]. However, the therapeutic effects of anti-PD-L1 mAband anti-PD-1 mAbare limited. Complete response (CR) or partial response (PR) of pembrolizumab and nivolumab in the treatment of cervical cancer Only $14 \%$ and $26.3 \%[8,9]$. Therefore, we need to find immunomodulators against other immune checkpoints to treat malignant tumors.

Yu et al. discovered TIGIT, a gene expressed on T and NK cells that encodes a protein with a variable immunoglobulin domain and an immunoreceptor tyrosine-based inhibitory motif (ITIM). TIGIT is an inhibitory receptor found primarily on NK cells, $\mathrm{CD} 8^{+} \mathrm{T}$ cells, $\mathrm{CD} 4^{+} \mathrm{T}$ cells, and regulatory $\mathrm{T}$ cells (Tregs) [10]. TIGIT is overexpressed in tumor-infected lymphocytes (TILs) from various solid tumors, including melanoma, Glioblastoma, non-small cell lung cancer, and liver cancer [11-14.]Furthermore, TIGIT is linked to poor cancer clinical outcomes. TIGIT expression in TILs in melanoma patients is associated with tumor metastasis [15], and $\mathrm{TIGIT}^{+} \mathrm{CD} 8^{+} \mathrm{T}$ cell expression in the PBMC of patients with gastric cancer is associated with poor survival [16]. TIGIT can suppress the immune system by binding to three ligands on tumor cells: CD155, CD112, and CD113. TIGIT has a high affinity for CD155 [10]. Our research results showed that TIGIT is expected to become a new target for the treatment of cervical cancer, and its blockade antibody alone or in combination with anti-PD-1/PD-L1 has significant effects in preclinical models of cervical cancer.

$\mathrm{TIGIT}^{+} \mathrm{CD}^{+}{ }^{+} \mathrm{T}$ cells were found to be significantly increased in cervical cancer patients, according to our findings. $\mathrm{TIGIT}^{+} \mathrm{CD} 8^{+} \mathrm{T}$ cells were functionally drained. $\mathrm{CD} 155$, which is expressed in cervical cancer cells, interacts with TIGIT and impairs CD8 ${ }^{+}$T cell effector function. Blocking the TIGIT/CD155 pathway improves $\mathrm{CD}^{+} \mathrm{T}$ cell effector function and slows tumor progression. This study identifies a possible therapeutic target for cervical cancer.After TIGIT/CD155 ligation, ITT-like motifs are phosphorylated and SH2-containing inositol phosphatase 1 (SHIP-1) is recruited, which prematurely terminates MAPK and NF$\mathrm{KB}$ signaling, leading to down-regulation $\mathrm{CD} 8^{+} \mathrm{T}$ cell function.

\section{Materials And Methods}

\section{Patients and tissue samples}

A total of 113 peripheral blood samples were collected, including 42 from cervical cancer patients, 35 high-grade squamous intraepithelial lesion (HSIL) patients, and 36 from subjects with normal cervix. In addition, we collected 11 pairs of cervical cancer and adjacent tissues. All the included cervical cancer patients underwent primary surgery. All samples were collected from the Qilu Hospital of Shandong University. The study was approved by Qilu Hospital's ethics committee.

\section{Cell Isolation}


Isolation of peripheral blood mononuclear cells (PBMC) from whole blood using Ficoll (TBD science, Tianjin, China) density gradient centrifugation. We obtained tumor single cell suspension from fresh tumor tissue. According to the manufacturer's instructions, a gentle MACS C tube (Milteny Biotec, Bergisch Gladbach, Germany) was used for mechanical dissociation and a tumor dissociation kit (Milteny Biotec) Enzymatic hydrolysis. The digested cells were filtered through a 70 $\mu \mathrm{m}$ mesh, centrifuged with Ficoll (Solarbio, Beijing), and the monocytes were resuspended in RPMI 1640.

\section{Cell Culture}

Mouse cervical cancer cell line U14 was obtained from the National Biomedical experimental cell resource bank (Beijing, China). U14 was cultured in DMEM supplemented with $10 \%$ fetal bovine serum (all from Gibco, Grand Island, NY, USA), $50 \mathrm{U} / \mathrm{mL}$ penicillin and $50 \mathrm{mg} / \mathrm{mL}$ streptomycin (all from Solarbio Science \& Technology, Beijing, China). $C D 8^{+} T$ cells were purified from PBMC positive selection by using a kit (Milteny Biotec, Bergisch Gladbach, Germany). CD8 ${ }^{+} T$ cells were stimulated with antiCD3/CD28(Stemcell, Canada) in T cell expansion medium (Stemcell, Canada). Activated CD8 ${ }^{+} T$ cells were treated with $5 \mu \mathrm{g} / \mathrm{mL}$ CD $155-\mathrm{Fc}, 10 \mu \mathrm{g} / \mathrm{mLCD} 155-\mathrm{Fc}$. Activated CD8 ${ }^{+} \mathrm{T}$ were cocultured with tumor cells at a 10:1 ratio.

Add $10 \mu \mathrm{g} / \mathrm{ml}$ anti-PD-1 mAb or $5 \mu \mathrm{g} / \mathrm{ml}$ anti-TIGIT mAb (R\&D Systems), respectively. As isotype control, we used a-human IgG1 (R\&D Systems). After 48 hours, $C D 8^{+} T$ cells were collected to determine cytokine production using the $T$ cell function assay.

\section{Flow Cytometry}

PBMCs isolated from cervical cancer patients or normal people were stained with fuorochrome conjugated PE-conjugated-anti-CD8 (Elabscence, Wuhan, China), PE-conjugated-anti-TIGIT-FITC (eBioscience) antibodies for $30 \mathrm{~min}$ in $4^{\circ} \mathrm{C}$. After washing 3 times, collect samples and use flow cytometry for detection. For intracellular staining, cell surface marker-stained cells were fixed and permeabilized with a fixation and permeabilization kit (BD Bioscience) for 20 minutes, and then treated with the fluorochrome-conjugated antibody APC-conjugated-anti-TNF-a (eBioscience), APC-conjugatedanti-IFN-y (eBioscience), APC-conjugated-anti-GramB (eBioscience) for intracellular staining at $4^{\circ} \mathrm{C}$ for 30 minutes. Finally, the stained cells were analyzed using a FACS Calibur flow cytometer (Becton Dickinson, USA), and the data were analyzed using Flow Jo software.

\section{Immuno histochemistry (IHC)}

For immunohistochemical analysis, the sections are deparaffinized, and then citric acid buffer is used for heat-mediated antigen retrieval. For testing, follow the manufacturer's instructions and use an immunohistochemistry detection kit (Zhongshan Jinqiao, Beijing, China). Sections were incubated at $4^{\circ} \mathrm{C}$ 
overnight with primary antibodies in PBS (anti-human TIGIT, 1:100, Cell Signaling Technology, Danvers, MA; anti-human CD155, 1:100, Cell Signaling Technology Danvers, MA, Abcam; anti-human CD8, Abcam, Cambridge, UK; anti-mouse CD8, 1:200, Cell Signaling Technology Danvers, MA). The slides were then incubated for 10 minutes at $37^{\circ} \mathrm{C}$ with biotin-labeled goat anti-rabbit lgG secondary antibody. Incubate the streptavidin peroxidase with the slides for 15 minutes at $37^{\circ} \mathrm{C}$ before staining with $\mathrm{DAB}$ (Zhongshan Jinqiao, Beijing, China). Meyer's hematoxylin (Solarbio Science \& Technology, Beijing, China) was used to stain the slides for 5 minutes. Seal the slices with neutral glue after they have been dehydrated.

\section{Multiplex Immunohistochemistry Staining (Mihc)}

For immunofluorescence analysis, we use multiple fluorescence immunohistochemical staining kits (Absin, Shanghai, China). Heat-mediated antigen retrieval and primary antibody incubation are the same as immunohistochemistry. After incubating the secondary antibody for 10 minutes, incubate the slides with the fluorescent staining amplification solution for 10 minutes at $37^{\circ} \mathrm{C}$. After washing three times with TBST, incubate the slides with 4',6-diamidino-2-phenylindole (DAPI) for 5 minutes. Finally, an antifluorescence quenching agent was used to seal the slides.

\section{Real-time Quantitative Rt-pcr (Qrt-pcr)}

Trizol reagent (Invitrogen) was used to extract total RNA from cells. After total RNA was quantified by spectrophotometry, reverse transcription was performed using the PrimeScript RT kit (Accurate biology, Hunan, China). Real-time PCR was performed using SYBR Premix Ex Taq (Accurate biology, Hunan, China) and 7900HT fast real-time PCR system (Applied Biosystems, Waltham, MA, USA). The primer sequences of genes including TIGIT, PD-1, LAG3, Tim3 and $\beta$-actin are shown in the supplementary information. The mRNA level of a specific gene were normalized with $\beta$-actin.

\section{Western Blot}

After washing the cells three times with PBS, they were lysed on ice in radioimmunoprecipitation analysis buffer (RIPA; Beyotime, China Institute of Biotechnology, 1\% phenylmethylsulfonyl fluoride (PMSF); $1 \%$ $\mathrm{NaF}$ ) for 30 minutes. Centrifuge at $12,000 \mathrm{rpm}$ for 10 minutes at $4^{\circ} \mathrm{C}$, and collect the supernatant. Next, the proteins were separated by SDS-PAGE and transferred to a PVDF membrane (Merck Millipore, Burlington, Massachusetts, USA). Incubate the membrane with the primary antibody $\beta$-actin $(1: 1000$, Cell Signaling Technology), TIGIT (1:1000, Cell Signaling Technology), SHIP-1 (1:1000, Cell Signaling Technology), ERK (1:1000, Cell Signaling Technology), p-ERK (1:1000, Cell Signaling Technology), p-IKBa (1:1000, Cell Signaling Technology), p-NF-KBP65 (1:1000, Cell Signaling Technology) overnight at $4^{\circ} \mathrm{C}$, and then incubate with the appropriate secondary antibody. ImageJ software (National Institutes of Health) was used to analyze relative protein levels, and $\beta$-actin was used as an endogenous control. 


\section{Immunoprecipitation}

The cells were placed in lysis buffer (Beyotime Biotechnology, China), lysed on ice for 30 minutes, and centrifuged at $15000 \mathrm{rpm}$ for 15 minutes at $4^{\circ} \mathrm{C}$. At $4^{\circ} \mathrm{C}$ overnight, incubate $10 \mathrm{mg}$ of antibody with 1000 $\mathrm{mg}$ of protein supernatant. Collect the supernatant and incubate it with Protein A/G Sepharose beads (Santa Cruz, USA) for 6 hours. The beads were washed three times and boiled before the immunoprecipitated protein was detected using western blotting.

\section{Cas9-sgrna Knockout}

\section{Cas9-sgRNA knockout}

Cas9 and single-guide RNA (sgRNAs) lentiviruses were designed and constructed by OBiO Technology Company (Shanghai, China). A lentivirus containing Cas 9 and sgRNA (sg-scramble, sg-CD155) was introduced into U14 cells. The sgRNA sequence used is ATTCGACAGGCGTCTTGGGAGGG. After 48 hours, the transfected cells were selected in a medium containing puromycin. Compared with the control group, the silencing efficiency of U14 cells is nearly $99 \%$.

\section{In Vivo Treatments}

Female C57BL6 mice (18-22g, 4-6 weeks old) were purchased from Beijing Vital River Laboratory Animal Technology Co., Ltd. for this study. U14 cells were trypsinized, resuspended in PBS, and inject $200 \mathrm{ml}$ $\left(1 \times 10^{7}\right.$ cells $)$ of the cell suspension into the subcutaneous area of the right armpit of each mice. For in vivo blockade, 3 days after injection of cell suspension, mice were randomly allocated to the anti-PD-1

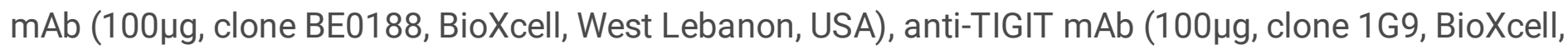
West Lebanon, USA), anti-TIGIT mAb + anti-PD-1 mAb and IgG (Mouse IgG1, clone MOPC-21, BioXcell) group. Three times a week intraperitoneal injection of blocking antibody and isotype control group. To

investigate the antitumor effects by target $C D 8^{+} T$ cells, mice were subcutaneously inoculated with $1 \times 10^{6}$ U14-NC-CD155 or U14-KO-CD155.

\section{Statistical Analysis And Bioinformatics}

GraphPad Prism 7.0 (GraphPad Software, San Diego, CA) was used for statistical significance analysis. The results are expressed as mean \pm standard deviation. The two-tailed Student's t-test was used for statistical comparison between two independent groups; a non-parametric test was used for data that does not conform to the normal distribution. Gene expression and clinical annotation data were downloaded from GEO (Gene Expression Omnibus) and TCGA (The Cancer Genome Atlas). The "limma" package was used to analyze diferentially expressed genes between cervical cancer and normal. For all experiments, a p-value less than 0.05 is the key to a significant difference ( $P$ value is expressed as: ${ }^{*} \mathrm{P}<$ $0.05, * \star P$ value $<0.01, * \star * P<0.001)$ 


\section{Results}

\section{TIGIT was highly expressed in cervical cancer patients}

To determine whether TIGIT is involved in cervical cancer progression, we analyzed the differential expression of TIGIT in cervical cancer and normal cervical tissues through the TCGA and GEO databases. The expression of TIGIT in cervical cancer patients was significantly higher than normal people (Fig. 1.A). We assessed PBMCs from 16 cervical cancer patients, $15 \mathrm{HSIL}$ patients, and 16 normal people for TIGIT expression. As shown in Fig. 1.B, the expression of TIGIT in cervical cancer patients was significantly higher than that of HSIL patients and normal people. Co-expression of immune checkpoint molecules may cause T lymphocyte exhaustion[17, 18]. Therefore, we tested the expression of TIGIT with coinhibitory receptor PD-1, lymphocyte activation gene 3 protein (LAG3), and T cell immunoglobulin and mucin domain-containing protein 3 (Tim3) in the PBMC of cervical cancer. We observed that TIGIT was positively correlated with PD-1, LAG3, Tim3 on $\mathrm{CD}^{+} \mathrm{T}$ cells from PBMC of patients with cervical cancer (Fig. 1. C, D, E). The immunohistochemical results of 11 pairs of cervical cancer and adjacent tissues showed that the number of TIGIT positive cells in cervical cancer tissues increased significantly (Fig. 1F). A quantitative analysis was shown in Supplementary Fig. 1A.

\section{Tigitcd8t Cells Was Highly Expressed In Cervical Cancer Patients}

We assessed PBMCs from 20 cervical cancer patients, 20 HSIL patients, and 20 normal people for TIGIT expression on $\mathrm{CD}^{+} \mathrm{T}$ cells. As shown in Fig. 2.A, the ratio of $\mathrm{TIGIT}^{+} \mathrm{CD} 8^{+} \mathrm{T}$ cells in cervical cancer patients and HSIL patients was significantly higher than that of normal people. We compared the functions of $\mathrm{TIGIT}^{+} \mathrm{CD} 8^{+} \mathrm{T}$ cells and $\mathrm{TIGIT}^{-} \mathrm{CD} 8^{+} \mathrm{T}$ cells in PBMC. The ability of $\mathrm{TIGIT}^{+} \mathrm{CD} 8^{+} \mathrm{T}$ cells to secrete cytokines (TNF- $a$ and IFN- $\gamma$ ) was significantly lower than that of TIGIT-CD8 ${ }^{+}$T cells (Fig. 2.C), which indicated that $\mathrm{TIGIT}^{+} \mathrm{CD} 8^{+} \mathrm{T}$ cells have low effector function and anti-tumor potential.

Immunohistochemical staining was subsequently used to investigate TIGIT expression in cervical cancer. Sections of cervical cancer tissues and adjacent cancers tissues were subjected to mIHC. TIGIT and $\mathrm{CD} 8^{+} \mathrm{T}$ cells were labeled for observation by laser confocal microscopy. We then evaluated the expression of $\mathrm{TIGIT}^{+} \mathrm{CD} 8^{+} \mathrm{T}$ cells through $\mathrm{mIHC}$. $\mathrm{TIGIT}^{+} \mathrm{CD} 8^{+} \mathrm{T}$ cells in cervical cancer tissues were significantly higher than those in para cancer (Fig. 2B). Quantitative analysis was shown in Supplementary Fig. 1B.

\section{Database analysis CD155 was highly expressed in cervical cancer patients}

CD155 is a high-affinity ligand for TIGIT [10]. Download the expression data of cervical cancer and normal cervical tissue through the TCGA and GEO databases, use the "limma package" to analyze differential gene expression, and standardize the data. The results showed that in the databases GSE29570, GSE52903, GSE67522 and TCGA-GTEx, the expression level of CD155 in cervical cancer 
tissues was higher than that in normal cervical tissues (Fig. 3. A, B). We conducted survival analysis on databases with survival information, and the results showed that in databases GSE44001, GSE52903 and TCGA-GTEx, cervical cancer patients with high expression of CD155 have shorter progression-free survival or overall survival. Our previous studies confirmed that CD155 expression was elevated in the tissues and plasma of patients with cervical cancer [19].

Next, we used the algorithm to study the cervical cancer database with a large sample size. In the GSE44001 and TCGA data sets, the expression of CD155 was negatively correlated with the level of CD8 ${ }^{+}$T cells (Fig. 3.C, D).

\section{TIGIT/CD155 blockade reverses the inhibition of $\mathrm{CD}^{+} \mathrm{T}$ cell cytokine production by cervical cancer cells}

Immunohistochemical analysis CD155 was expressed in cervical cancer and negatively correlated with CD8 ${ }^{+} T$ cells (Fig. 4A, B). We investigated whether CD155 was involved in mediating $C D 8^{+}{ }^{\top}$ cell function inhibition. $C D 8^{+} T$ cells were sorted from the PBMC of patients with cervical cancer. Activated $C D 8^{+} T$ cells were treated with $5 \mu \mathrm{g} / \mathrm{ml} \mathrm{CD} 155-\mathrm{Fc}, 10 \mu \mathrm{g} / \mathrm{ml} \mathrm{CD} 155-\mathrm{Fc}$, respectively. CD8 ${ }^{+} \mathrm{T}$ cells were collected after $24 \mathrm{~h}$ to determine cytokine production using the $T$ cell function assay. The high concentration of Fc-CD155 decreased the ability of $C D 8^{+} T$ cells to secrete cytokines IFN- $\gamma$, TNF- $a$ and GranzymeB (Fig. 4C). Quantitative analysis was shown in Supplementary Fig. 2A. CD155 can interact with $\mathrm{CD}^{+}{ }^{+} \mathrm{T}$ cells and affect CD $8^{+}$T cell function through CD155/TIGIT.

TIGIT is associated with T cell depletion. We next investigated whether TIGIT blockade can affect the effector function of T cells. The TIGIT blocking antibody was used to block the TIGIT signal. CD ${ }^{+}{ }^{+}$cells were stimulated with aCD3/CD28 and cocultured with tumor cells in the presence of an anti-TIGIT blocking antibody or isotype control. Our results showed that inhibiting CD8 ${ }^{+} \mathrm{T}$ cell production of IFN- $\mathrm{y}$, TNF-a and GranzymeB were reversed after blocking TIGIT (Fig. 4.D). A study showed that exhausted CD8 ${ }^{+} T$ cells coexpress TIGIT and PD-1 [17]. We also investigated whether TIGIT and PD-1 synergistically affect T cell effector functions. Blocking TIGIT or PD-1 increased the production of IFN- $\gamma$, TNF- $a$ and GranzymeB in $\mathrm{CD}^{+}{ }^{+} \mathrm{T}$ cells. Blocking both TIGIT and PD-1 further enhanced IFN- $\gamma$, TNF- $\alpha$ and GranzymeB production in $\mathrm{CD}^{+} \mathrm{T}$ cells (Fig. 4.D). Quantitative analysis was shown in Supplementary Fig. 2B. This suggested that TIGIT and PD-1 work together to induce CD8 ${ }^{+} T$ cell exhaustion.

\section{TIGIT/CD155 signaling inhibited the activation of the NF-KB and ERK pathways}

$\mathrm{NF}-\mathrm{KB}$ is a critical transcription factor that controls the production of various cytokines in various immune cells [20]. Previous studies have shown that mitogen-activated protein kinase (MAPK) and nuclear factorKB (NF-KB) signaling pathways play an essential role in TIGIT/CD155-mediated immunosuppressive effects on NK cells [21]. Next, we analyzed the changes of related proteins in CD8 ${ }^{+} T$ cells after incubation with anti-TIGIT mAb and Fc-CD155. Blocking TIGIT during the culture of activated T cells significantly increased the ratio of $p$-ERK/ERK, p-IKBa and p-NF-KBP65 levels. In addition, the ratio of p-ERK/ERK, pIKBa and p-NF-KBP65 were significantly lower in the CD8 ${ }^{+}$T cells cultured with Fc-CD155 (Fig. 5A). SHIP-1 
is associated with negative regulation of T cell activation [21]. Early experiments have shown that the connection of CD3 or CD28 on T cells leads to the phosphorylation and catalytic activation of SHIP-1, indicating the role of SHIP-1 in lymphocyte activation [22]. Silencing SHIP-1 in CD $4^{+} T$ lymphocytes leads to the loss of $p$-ERK protein and reduces the proliferation and movement of CD $4^{+} T$ cells [23]. In our study, blocking TIGIT during the culture of activated T cells reduced SHIP-1 expression. Blocking both TIGIT and PD-1 further decreased SHIP-1. Activated CD8 ${ }^{+}$T cells cultured with Fc-CD155, SHIP expression was significantly increased.

In order to explore the potential mechanism of TIGIT/CD155 inhibiting the function of CD8 ${ }^{+} \mathrm{T}$ cells, we performed immunoprecipitation analysis on $\mathrm{CD}^{+} \mathrm{T}$ cells cultured with Fc-CD155. We performed coimmunoprecipitation (Co-IP) analysis and observed that TIGIT could precipitate SHIP-1 under the action of Fc-CD155 (Fig. 5B).

\section{Targeting Tigit/cd155 To Inhibited Tumor Progression In Vivo}

To further verify the antitumor effects of targeting TIGIT/CD155 signaling in vivo, we constructed tumor xenograft models by injecting C57BL6 mice with U14 cells transfected with WT (Wild Type) U14, U14-NCCD155 or U14-KO-CD155. Compared with the control group, the silencing efficiency of U14 cells is nearly 99\% ((Fig. 6A). Mice injected with WT-U14 cells were treated with PD-1 blocking antibodies, TIGIT blocking antibodies or isotype control.

Tumor progression was inhibited in mice that received U14- KO-CD155 (Fig. 6B). The U14- KO-CD155 group tumor tissue $C D 8^{+} T$ lymphocyte infiltration level and $C D 8^{+} T$ lymphocyte secretion function factors TNF- $\alpha$ and IFN- $y$ levels increased (Fig. 6C, F). Quantitative analysis was shown in Supplementary Fig. 3A, C, D.

In WT-U14 C57BL/6 mice, the blocking TIGIT group inhibited the growth and proliferation of cervical cancer transplanted C57BL/6 mice (Fig. 6D). The combined blocking of TIGIT and PD-1 group further inhibited C57BL/ 6 than the blocking TIGIT group alone. In addition, the combined blocking of TIGIT and PD-1 group tumor tissue $C D 8^{+} T$ lymphocyte infiltration level and $C D 8^{+} T$ lymphocyte secretion function factors TNF- $a$ and IFN- $y$ levels further increased compared with the single blocking TIGIT group (Fig. 6E, G). Quantitative analysis was shown in Supplementary Fig. 3B, E, F.

\section{Discussion}

Immunotherapy has joined the ranks of surgery, radiotherapy, chemotherapy, and targeted therapy and has become one of the methods of cancer treatment [24]. Immune escape is a defect in the immune system that promotes carcinogenesis. When naive $T$ cells are activated and differentiate into effector $T$ cells within 1-2 weeks, it is accompanied by the acquisition of robust proliferation, transcription, 
epigenetics, metabolic reprogramming, and effector T cell characteristics. In this development process, the gradual loss of effector functions, the continuous up-regulation and co-expression of multiple inhibitory receptors, and the expression of key transcription factors all affect the differentiation state of $T$ lymphocytes and lead to T cell depletion [25]. T cell exhaustion is promoted by activating of inhibitory immune checkpoints (such as PD-1 and CTLA-4) [20]. TIGIT/CD155 has attracted widespread attention as a new immune checkpoint and a potential target for cancer immunotherapy. TIGIT is the first to identify inhibitory receptors that exert immunosuppressive effects through activated $\mathrm{CD} 4^{+} \mathrm{T}$ cells, Tregs and NK cells [10][21, 22]. The study on the immunosuppressive effect of TIGIT on CD8 ${ }^{+} T$ is increasing. The role and mechanism of TIGIT/CD155 in cervical cancer have not been studied. Our study showed that TIGIT was upregulated in CD8 ${ }^{+} T$ cells infiltrated by cervical cancer patients. Compared with normal people, the expression of $\mathrm{TIGIT}^{+} \mathrm{CD} 8^{+} \mathrm{T}$ cells in PBMC and cervical cancer tissues increased significantly. CD155 is the ligand of TIGIT and can interact with TIGIT with high affinity [10]. Loss of host and tumorderived CD155 reduces tumor growth and metastasis and increases response to immunotherapy [17][23] [26]. Data analysis of the TCGA and GEO databases showed that the expression level of CD155 in cervical cancer tumor tissue was higher than that in normal tissue, and it was related to the shorter survival of cervical cancer patients. Our previous studies were consistent with that, CD155 is highly expressed in the plasma and tissues of patients with cervical cancer and is related to the progression of cervical cancer [19]. In our studies, the blockade of TIGIT enhanced the secretion of cytokines. However, TIGIT levels were significantly upregulated on $C D 8^{+} T$ cells cultured with Fc-CD155. The activation of TIGIT/CD155 signal inhibited the immune function of $C D 8^{+} T$ cells.

TIGIT contains ITT-like phosphorylation motifs and non-canonical ITIM motifs in the cytoplasmic tail [27]. Receptors containing inhibitory ITIM usually undergo cytoplasmic tail tyrosine phosphorylation in the first step of negative signal transduction [28]. Then, they recruit SH2 domain containing tyrosine phosphatase (such as SHP1 and SHP2) or inositol phosphatase (such as SHIP-1 and SHIP-2) to induce inhibitory signaling [29]. Studies on the effect of SHIP on macrophage phagocytosis have shown that SHIP expression down-regulates Rac activity and subsequent superoxide production [30]. Previous studies have shown that TIGIT-mediated recruitment of SHIP-1 inhibits the NK cell pathway by blocking PI3K and MAPK signaling [31]. NF-KB protein can regulate the expression of hundreds of genes, and regulate important physiological processes such as inflammation, immunity, proliferation, and cell death [32]. The NF-KB pathway regulates the development of $T$ cells and activates TCR signals to trigger $T$ cell functions [33]. We showed that phosphorylated TIGIT binds to CD155 to inhibit NF-KB and ERK activation by recruiting SHIP-1, leading to down-regulation of cytokine production. Inactivated CD $8^{+} T$ cells, after blocking TIGIT, the inhibitory effect of SHIP-1 on CD8 ${ }^{+} T$ cells is weakened, and the activation of NF-KB and ERK is enhanced.

Co-expression of TIGIT and other immune checkpoints can lead to $\mathrm{CD}^{+}{ }^{+} \mathrm{T}$ cell depletion $[17,18]$. Blocking of TIGIT and PD-1 increased cytokine production. We confirmed that the combined blockade of TIGIT and PD-1 further enhanced the immune response in tumor-bearing mice and tumor suppression compared 
with targeting either one (Fig. 7). This suggests that combining PD-1 and TIGIT inhibition could treat cervical cancer.

\section{Conclusion}

In summary, the TIGIT/CD155 signaling pathway has enhanced effects in cervical cancer patients and mouse models and is related to immunosuppression. Targeting the TIGIT/CD155 signaling pathway may be a potential therapeutic strategy for the treatment of cervical cancer.

\section{Declarations}

\section{Ethics approval and consent to participate}

This study was approved by the Ethics Committee of the Qilu Hospital of Shandong University. All participants were recruited after providing signed informed consent.

\section{Consent for publication}

Not available.

\section{Availability of data and materials}

The data used and/or analysis during the current study are available from the corresponding author on reasonable request.

\section{Competing interests}

No potential conflicts of interest to disclose.

\section{Funding}

This work was supported by the the National Natural Science Foundation of China (NSFC, 81572559). The Key Research Project of Shandong Province (2017CXGC1210). Jinan City "20 New Universities" independent innovation group (2021GXRC027).

\section{Authors' contributions}

YZ: Conceptualization, Methodology, LL: Writing-Original Draft and Investigation, AWang: Methodology, XL: Methodology, SH: Visualization, Investigation, YS: Software, Validation, JZ: Validation , LG: Validation and Editing.

\section{Acknowledgements}

We sincerely appreciate all participants in the study. 


\section{References}

1. Torre L, Islami F, Siegel R, Ward E, Jemal A: Global Cancer in Women: Burden and Trends. Cancer epidemiology, biomarkers \&prevention : a publication of the American Association for Cancer Research, cosponsored by the American Society of Preventive Oncology 2017, 26(4):444-457.

2. Bray F, Ferlay J, Soerjomataram I, Siegel R, Torre L, Jemal A: Global cancer statistics 2018 : GLOBOCAN estimates of incidence and mortality worldwide for 36 cancers in 185 countries. CA Cancer J Clin 2018, 68(6):394-424.

3. Canfell K: Towards the global elimination of cervical cancer. Papillomavirus research (Amsterdam, Netherlands) 2019, 8:100170.

4. Drakes M, Czerlanis C, Stiff P: Immune Checkpoint Blockade in Gynecologic Cancers: State of Affairs. Cancers (Basel) 2020, 12(11).

5. Willimsky G, Blankenstein T: Sporadic immunogenic tumours avoid destruction by inducing T-cell tolerance. Nature 2005, 437(7055):141-146.

6. Pardoll D: The blockade of immune checkpoints in cancer immunotherapy. Nat Rev Cancer 2012, 12(4):252-264.

7. Topalian S, Drake C, Pardoll D: Immune checkpoint blockade: a common denominator approach to cancer therapy. Cancer Cell 2015, 27(4):450-461.

8. Yang W, Song Y, Lu Y, Sun J, Wang H: Increased expression of programmed death (PD)-1 and its ligand PD-L1 correlates with impaired cell-mediated immunity in high-risk human papillomavirusrelated cervical intraepithelial neoplasia. Immunology 2013, 139(4):513-522.

9. Chung H, Ros W, Delord J, Perets R, Italiano A, Shapira-Frommer R, Manzuk L, Piha-Paul S, Xu L, Zeigenfuss $S$ et al: Efficacy and Safety of Pembrolizumab in Previously Treated Advanced Cervical Cancer: Results From the Phase II KEYNOTE-158 Study. Journal of clinical oncology : official journal of the American Society of Clinical Oncology 2019, 37(17):1470-1478.

10. Yu X, Harden K, Gonzalez L, Francesco M, Chiang E, Irving B, Tom I, Ivelja S, Refino C, Clark H et al: The surface protein TIGIT suppresses T cell activation by promoting the generation of mature immunoregulatory dendritic cells. Nature immunology 2009, 10(1):48-57.

11. Chauvin J, Pagliano O, Fourcade J, Sun Z, Wang H, Sander C, Kirkwood J, Chen T, Maurer M, Korman A et al: TIGIT and PD-1 impair tumor antigen-specific CD8 8 T cells in melanoma patients. J Clin Invest 2015, 125(5):2046-2058.

12. O'Brien S, Klampatsa A, Thompson J, Martinez M, Hwang W, Rao A, Standalick J, Kim S, Cantu E, Litzky L et al: Function of Human Tumor-Infiltrating Lymphocytes in Early-Stage Non-Small Cell Lung Cancer. Cancer Immunol Res 2019, 7(6):896-909.

13. Raphael I, Kumar R, McCarl L, Shoger K, Wang L, Sandlesh P, Sneiderman C, Allen J, Zhai S, Campagna $\mathrm{M}$ et al: TIGIT and PD-1 Immune Checkpoint Pathways Are Associated With Patient Outcome and Anti-Tumor Immunity in Glioblastoma. Front Immunol 2021, 12:637146. 
14. Stålhammar G, Seregard S, Grossniklaus H: Expression of immune checkpoint receptors Indoleamine 2,3-dioxygenase and T cell Ig and ITIM domain in metastatic versus nonmetastatic choroidal melanoma. Cancer Med 2019, 8(6):2784-2792.

15. Zhang C, Wang Y, Xun X, Wang S, Xiang X, Hu S, Cheng Q, Guo J, Li Z, Zhu J: TIGIT Can Exert Immunosuppressive Effects on CD8+ T Cells by the CD155/TIGIT Signaling Pathway for Hepatocellular Carcinoma In Vitro. Journal of immunotherapy (Hagerstown, Md : 1997) 2020, 43(8):236-243.

16. Tang W, Pan X, Han D, Rong D, Zhang M, Yang L, Ying J, Guan H, Chen Z, Wang X: Clinical significance of CD8 T cell immunoreceptor with Ig and ITIM domains in locally advanced gastric cancer treated with SOX regimen after D2 gastrectomy. Oncoimmunology 2019, 8(6):e1593807.

17. He W, Zhang H, Han F, Chen X, Lin R, Wang W, Qiu H, Zhuang Z, Liao Q, Zhang W et al: CD155T/TIGIT Signaling Regulates CD8+T-cell Metabolism and Promotes Tumor Progression in Human Gastric Cancer. Cancer Res 2017, 77(22):6375-6388.

18. Inozume T, Yaguchi T, Furuta J, Harada K, Kawakami Y, Shimada S: Melanoma Cells Control Antimelanoma CTL Responses via Interaction between TIGIT and CD155 in the Effector Phase. J Invest Dermatol 2016, 136(1):255-263.

19. Liu L, Wang Y, Geng C, Wang A, Han S, You X, Sun Y, Zhang J, Lu W, Zhang Y: CD155 Promotes the Progression of Cervical Cancer Cells Through AKT/mTOR and NF-KB Pathways. Frontiers in oncology 2021, 11:655302.

20. Chen L: Co-inhibitory molecules of the B7-CD28 family in the control of T-cell immunity. Nat Rev Immunol 2004, 4(5):336-347.

21. Boles K, Vermi W, Facchetti F, Fuchs A, Wilson T, Diacovo T, Cella M, Colonna M: A novel molecular interaction for the adhesion of follicular CD4 T cells to follicular DC. Eur J Immunol 2009, 39(3):695703.

22. Stanietsky N, Simic H, Arapovic J, Toporik A, Levy O, Novik A, Levine Z, Beiman M, Dassa L, Achdout $\mathrm{H}$ et al: The interaction of TIGIT with PVR and PVRL2 inhibits human NK cell cytotoxicity. Proc Natl Acad Sci USA 2009, 106(42):17858-17863.

23. Li X, Das I, Lepletier A, Addala V, Bald T, Stannard K, Barkauskas D, Liu J, Aguilera A, Takeda K et al: CD155 loss enhances tumor suppression via combined host and tumor-intrinsic mechanisms. J Clin Invest 2018, 128(6):2613-2625.

24. Wherry E, Kurachi M: Molecular and cellular insights into T cell exhaustion. Nat Rev Immunol 2015, 15(8):486-499.

25. Sharma P, Allison J: The future of immune checkpoint therapy. Science 2015, 348(6230):56-61.

26. Wu L, Mao L, Liu J, Chen L, Yu G, Yang L, Wu H, Bu L, Kulkarni A, Zhang W et al: Blockade of TIGIT/CD155 Signaling Reverses T-cell Exhaustion and Enhances Antitumor Capability in Head and Neck Squamous Cell Carcinoma. Cancer Immunol Res 2019, 7(10):1700-1713.

27. Engels $\mathrm{N}$, Wienands $\mathrm{J}$ : The signaling tool box for tyrosine-based costimulation of lymphocytes. Current opinion in immunology 2011, 23(3):324-329. 
28. Lanier L: Up on the tightrope: natural killer cell activation and inhibition. Nature immunology 2008, 9(5):495-502.

29. Kerr W, Colucci F: Inositol phospholipid signaling and the biology of natural killer cells. Journal of innate immunity 2011, 3(3):249-257.

30. Harris SJ, Parry RV, Foster JG, Blunt MD, Wang A, Marelli-Berg F, Westwick J, Ward SG: Evidence that the lipid phosphatase SHIP-1 regulates T lymphocyte morphology and motility. J Immunol 2011, 186(8):4936-4945.

31. Liu S, Zhang H, Li M, Hu D, Li C, Ge B, Jin B, Fan Z: Recruitment of Grb2 and SHIP1 by the ITT-like motif of TIGIT suppresses granule polarization and cytotoxicity of NK cells. Cell death and differentiation 2013, 20(3):456-464.

32. Zinatizadeh M, Schock B, Chalbatani G, Zarandi P, Jalali S, Miri S: The Nuclear Factor Kappa B (NFkB) signaling in cancer development and immune diseases. Genes \& diseases 2021, 8(3):287-297.

33. Blanchett S, Boal-Carvalho I, Layzell S, Seddon B: NF-kB and Extrinsic Cell Death Pathways Entwined Do-or-Die Decisions for T cells. Trends in immunology 2021, 42(1):76-88.

\section{Figures}

\section{Figure 1}

The expression level of TIGIT in cervical cancer patients. (A)Analyze the expression levels of cervical cancer tissues and normal cervical tissues in GSE29570, GSE52903, GSE67522 and TCGA-GTEx databases. (B) The expression level of TIGIT in PBMC of cervical cancer patients, HSIL patients and normal cervix.(C, D, E) Correlation analysis of TIGIT and PD-1, TIGIT and LAG3, TIGIT and Tim3 in PBMC of patients with cervical cancer. $(\mathrm{F})$ The expression level of $\mathrm{TIGIT}^{+}$cells in cervical cancer tissues and paracancer.

\section{Figure 2}

The expression level of $\mathrm{TIGIT}^{+} \mathrm{CD} 8^{+} \mathrm{T}$ cells in cervical cancer patients was related to $\mathrm{CD} 8^{+} \mathrm{T}$ cell depletion. (A) The proportion of $\mathrm{TIGIT}^{+} \mathrm{CD} 8^{+} \mathrm{T}$ lymphocytes gradually increased in normal cervix, HSIL, and cervical cancer patients. (B) The expression of TIGIT in $\mathrm{CD} 8^{+} \mathrm{T}$ cells through mIHC. The expression level of $\mathrm{TIGIT}^{+} \mathrm{CD} 8^{+} \mathrm{T}$ cells in cervical cancer tissues and paracancer. (C) The levels of cytokines TNF-a and IFN- $y$ are secreted by $\mathrm{TIGIT}^{+} \mathrm{CD} 8^{+} \mathrm{T}$ cells and $\mathrm{TIGIT}^{-} \mathrm{CD} 8^{+} \mathrm{T}$ cells.

\section{Figure 3}


The expression level of CD155 in cervical cancer tissues. A correlation analysis between the expression level of CD155 and the level of CD8 ${ }^{+}$T cell infiltration. (A) GSE29570, GSE52903, GSE67522 and TCGAGTEx databases to analyze the expression level of CD155 mRNA in cervical cancer tissues. (B) GSE44001, GSE52903, and TCGA database based on Kaplan-Meier survival analysis results showed that patients with high CD155 expression have shorter progression-free survival or overall survival (GSE44001: $n=300$; GSE52903: $n=55$; TCGA: $n=304$ ). (C) Correlation analysis between the expression level of CD155 and the level of $C D 8^{+} T$ cell infiltration in TCGA and GSE44001 with ESTIMATE score, CIBERSORT, and XCELL algorithm.

\section{Figure 4}

$(A, B)$ The expression level of CD155 in cervical cancer patients was related to $C D 8^{+} T$ cell depletion. (C)Activated $\mathrm{CD} 8^{+} \mathrm{T}$ cells were treated with $\mathrm{CD} 155-\mathrm{Fc}$. The ability of $\mathrm{CD} 8^{+} \mathrm{T}$ cells to secrete cytokines IFN- $\mathrm{Y}$, TNF-a and GranzymeB. (D)CD8+T cells were stimulated with aCD3/CD28 and cocultured with tumor cells in the presence of an anti-TIGIT, anti-PD-1 blocking antibody or isotype control.The production of IFN- $y$, TNF-a and GranzymeB in CD8 ${ }^{+} T$ cells.

\section{Figure 5}

TIGIT/CD155 signaling suppresses NF-KB and ERK pathway activation.

(A) Activated $\mathrm{CD} 8^{+} \mathrm{T}$ cellswere stimulated with aCD3/CD28 and cocultured with tumor cellsin the presence of an anti-TIGIT blocking antibody, anti-PD-1 blocking antibody.Activated CD8 ${ }^{+} \mathrm{T}$ cells were treated with CD155-Fc. Expression levels of p-ERK/ERK, p-IkBa and p-NF-kBP65.(B) SHIP-1 was associated with negative regulation of $\mathrm{T}$ cell activation. We performed Co-IP analysis and observed that TIGIT could precipitate SHIP-1 under the action of Fc-CD155.

\section{Figure 6}

TIGIT/CD155 inhibited the growth of cervical cancer transplanted tumors and the function of CD8 ${ }^{+} T$ lymphocytes in C57BL/6 mice.(A) Compared with the control group, the CD155 knockout efficiency of U14 cells. (B) Analysis of tumor progression in mice that received U14-NC-CD155 and U14-KO-CD155. (C) IHC analysis of CD8 ${ }^{+} \mathrm{T}$ cell infiltration level in tumors in mice received U14-NC-CD155 and U14-KOCD155. (D) WT-U14 cells were treated with PD-1 blocking antibodies, TIGIT blocking antibodies or isotype control. Analysis of tumor progression in mice that receivedtreatment. (E)CD8 ${ }^{+} T$ cells were isolated from mouse tumor tissues. IHC analysis of $\mathrm{CD}^{+} \mathrm{T}$ cell infiltration level in tumors in mice receivedtreatment. 
(F)CD ${ }^{+} T$ cells were isolated from mouse tumor tissues. The TNF- $\alpha$ and IFN- $y$ secreted by $C D 8^{+} T$ cells in mice received U14-NC-CD155 and U14-KO-CD155. (G)The TNF- $\mathrm{a}$ and IFN- $\gamma$ secreted by $\mathrm{CD}^{+}{ }^{+} \mathrm{T}$ cells in mice received treatment.

\section{Image not available with this version}

Figure 7

Schematic diagram of CD155/TIGIT binding to regulate CD8+ T cell function and related mechanisms.

\section{Supplementary Files}

This is a list of supplementary files associated with this preprint. Click to download.

- sFig1.tif

- sFig.2.jpg

- sFig3.tif 